\title{
Mega randomized clinical trials: a definitive solution or a double-edged sword?
}

\author{
Florian Lemaitre ${ }^{1,2,3}$ (1) $\cdot$ Bruno Laviolle $e^{1,2}$
}

Received: 6 August 2021 / Accepted: 7 October 2021 / Published online: 14 October 2021

(c) The Author(s), under exclusive licence to Springer-Verlag GmbH Germany, part of Springer Nature 2021

The severe acute respiratory syndrome coronavirus 2 (SARS-CoV-2) pandemic has hit the world from late 2019 leading to a rapid worldwide diffusion. The high number of patients hospitalized in conventional or in intensive care units as well as the prolonged hospital stay of patients threatened healthcare organization in numbers of countries. In addition, the infection fatality rate of SARS-CoV-2 infection prompted the implementation of non-pharmacological interventions going from social distancing to national lockdowns and stimulated the clinical research field. Several drugs used in other indications (antiviral, anti-inflammatory, or other pharmacological classes of drugs) were proposed as potential preventive or curative treatments [1]. Besides uncoordinated local initiatives and observational studies, mega randomized clinical trials (RCT) emerged as an appealing approach for SARS-CoV-2 treatments evaluation. During early 2020, two mega RCTs tackling drug efficacy on SARS-CoV-2 infection started: the World Health Organization (WHO) Solidarity study (ISRCTN83971151) and the United Kingdom University of Oxford Recovery trial (NCT04381936). The WHO Solidarity study evaluated remdesivir, a treatment developed for Ebola infection; lopinavir/ ritonavir with or without interferon beta, an association used in human immunodeficiency virus infection; and hydroxychloroquine, an immunomodulary drug used in the lupus treatment. The Recovery trial initially evaluated lopinavir/ ritonavir, hydroxychloroquine, azithromycin, a macrolide antibiotic, and dexamethasone, a corticosteroid drug. They

Florian Lemaitre

florian.lemaitre@chu-rennes.fr

1 Univ Rennes, CHU Rennes, Inserm, EHESP, Irset (Institut de recherche en santé, environnement et travail), UMR_S 1085, 35000 Rennes, France

2 INSERM, Centre d'Investigation Clinique, CIC 1414, F-35000 Rennes, France

3 Department of Pharmacology, Rennes University Hospital, 2 rue Henri Le Guilloux, 35033 Rennes Cedex, France combined an adaptative (the recovery trial has therefore added other treatment arms since its beginning), randomized design aiming at including drug candidates as they surfaced and a highly effective patient inclusion process leading to highly powered studies. By focusing every national and/or supranational health/research centers effort on a single study, this kind of RCT offers the possibility of an early answer to therapeutic hypotheses. This also limits the potential research waste elicited by low power/low rationale studies and avoids patient exposure to ineffective treatments [2]. Consequences of inappropriate drug use in patients during the recent pandemic included adverse events leading to morbidity and possibly excess mortality and antimicrobial resistance onset. Hence, mega-RCT initiative appears as the new gold standard in clinical research.

However, the coordination of such studies on a large scale should not be underestimated. They required, therefore, bringing together scientific leaders, regulatory agencies, and political commitment. Not to mention that, apart from these pre-requites, mega RCTs are not without any flaws as highlighted by the Recovery and Solidarity studies. First, constraining drugs' evaluation to a handful may decrease the chance to identify an effective treatment. Such an approach may also limit the scientific emulation between research teams leading to a decrease in the rate of scientific discovery. Then, designing mega RCTs did not prevent Recovery and Solidarity studies to evaluate drugs with very low pharmacological rationale, for efficacy on SARS-CoV-2 infection. For example, the two studies evaluated hydroxychloroquine and Recovery evaluated azithromycin without any convincing evidence of antiviral efficacy [3-5]. The pharmacokinetic profile of the drugs has raised concerns that they would not be able to exert virological response because of inability to reach in patients the targets identified in vitro. Moreover, at the time of the implementation of these drugs in the RCTs (i.e., March 1, 2020, and March 19, 2020, for Solidarity and Recovery, respectively), no appropriate pre-clinical data, and particularly no SARS-CoV-2 animal models, were available. Consequently, thousands of patients 
were exposed to these drugs while the minimum standard for drug selection into clinical trial was entirely reached. The negative results for these two treatment arms as well as other negative results coming from other RCTs have later confirmed the insufficiency of scientific background behind the therapeutic hypotheses. The story for drugs with antiinflammatory mechanism is different. While no appropriate pre-clinical model existed and given the pharmacological rationale supporting their use during the inflammatory phase of the SARS-CoV-2 infection, their selection as candidates for RCTs appeared to be legitimate. The reduction of patient mortality associated with the use of dexamethasone and tocilizumab in Recovery was a major success for the world scientific community [6,7]. While colchicine, another potential treatment but with a questionable safety profile, has been recently shown to be unable to decrease 28-days mortality, this should not depreciate the milestone reached by megaRCT during the pandemic [8].

Recent effort for mutualizing research resources should also be highlighted. France has created the CAPNET (Comite Ad-hoc de Pilotage National des Essais Thérapeutiques et autres recherches sur la COVID-19) national agency to give projects a "national research program priority" label aiming at fast tracking their implementation. However, this national initiative has no regulatory competencies that could allow combining clinical trials with the similar aim. Regarding vaccines, the COVIREIVAC platform was designed to promote clinical research on COVID-19 vaccines by mutualizing the effort of 32 regional hospital centers in France [9].

However, despite their evident virtues, mega RCTs can then still be associated with research waste and also present limitations, which should not be underestimated. A wellbalanced strategy aiming at mutualizing efforts without discouraging original initiatives has still to be proposed and, considering therapeutic RCTs, should aggregate the expertise of pharmacologists for drug candidates' selection, clinicians able to widely federate patient inclusion, and methodologists for the appropriate design and conduction of studies implementation. A strong scientific regulation on a national or ideally supra-national level is required to channel the clinical trial landscape with a powerful willingness to stop the projects with insufficient quality standard and to merge trials with similar or close objectives.

Author contribution FL and BL wrote the manuscript and gave their final approval for manuscript submission.
Availability of data and material Not applicable.

Code availability Not applicable.

\section{Declarations}

Ethics approval Not applicable.

Consent to participate Not applicable.

Consent for publication Not applicable.

Competing interests The author declares no competing interests.

\section{References}

1. Zeitlinger M, Koch BCP, Bruggemann R et al (2020) Pharmacokinetics/pharmacodynamics of antiviral agents used to treat SARS-CoV-2 and their potential interaction with drugs and other supportive measures: a comprehensive review by the PK/PD of Anti-Infectives Study Group of the European Society of Antimicrobial Agents. Clin Pharmacokinet 59:1195-1216. https://doi. org/10.1007/s40262-020-00924-9

2. Lemaitre F, Locher C, Verdier MC, Naudet F (2021) Clinical trials during pandemics and beyond: time for a more efficient pharmacological strategy. J Antimicrob Chemother dkab190. https://doi. org/10.1093/jac/dkab190

3. Horby P, Mafham M et al (2020) Effect of Hydroxychloroquine in Hospitalized Patients with Covid-19. RECOVERY Collaborative Group. N Engl J Med 383:2030-2040. https://doi.org/10.1056/ NEJMoa2022926

4. RECOVERY Collaborative Group (2021) Azithromycin in patients admitted to hospital with COVID-19 (RECOVERY): a randomised, controlled, open-label, platform trial. Lancet 397:605-612. https://doi.org/10.1016/S0140-6736(21)00149-5

5. Pan H, Peto R et al (2020) Repurposed antiviral drugs for Covid-19 - Interim WHO Solidarity Trial Results. WHO Solidarity Trial Consortium. N Engl J Med. https://doi.org/10.1056/NEJMoa2023184

6. Horby P, Lim WS et al (2021) RECOVERY Collaborative Group. Dexamethasone in hospitalized patients with Covid-19. N Engl J Med 384:693-704. https://doi.org/10.1056/NEJMoa2021436

7. RECOVERY Collaborative Group (2021) Tocilizumab in patients admitted to hospital with COVID-19 (RECOVERY): a randomised, controlled, open-label, platform trial. Lancet 397:1637-1645. https:// doi.org/10.1016/S0140-6736(21)00676-0

8. Group RC, Horby PW, Campbell M, et al (2021) Colchicine in patients admitted to hospital with COVID-19 (RECOVERY): a randomised, controlled, open-label, platform trial. medRxiv 2021.05.18.21257267. https://doi.org/10.1101/2021.05.18.21257267

9. Covireivac. https://www.covireivac.fr/. Accessed 7 Jul 2021

Publisher's Note Springer Nature remains neutral with regard to jurisdictional claims in published maps and institutional affiliations. 